\title{
ATLAS QCD measurements for Higgs studies and New Physics searches
}

\author{
Manuel Proissl ${ }^{1}$,a on behalf of the ATLAS collaboration \\ ${ }^{1}$ University of Edinburgh
}

\begin{abstract}
Our understanding of the modelling of Standard Model processes plays an important role for Higgs physics and searches beyond the Standard Model. Measurements such as vector boson plus jet production are essential for the understanding of backgrounds, modelling of double parton interactions, heavy flavour production from gluon splitting and modelling of the underlying event. Precision QCD measurements can also be used to constrain parton density functions, which are important for the understanding of the Higgs boson production cross section and the Standard Model backgrounds at the LHC kinematic limits. A review of recent ATLAS measurements is presented.
\end{abstract}

\section{Introduction}

A deep understanding of the phenomenology of Quantum Chromodynamics (QCD) at the LHC energy frontier is a fundamental prerequisite for the prediction of the abundant QCD backgrounds in Higgs boson analyses and searches for any significant deviations of our Standard Model expectations. Precision measurements of the production of jets, photons, their combination or vector boson plus jets are crucial tests of perturbative QCD predictions and provide important constraints on parton distribution functions (PDFs) of the proton. For instance, these constraints can significantly reduce the gluon PDF uncertainty, which presently dominates theory errors in studies of the Higgs boson production via the gluon fusion process, and thus have a direct impact on our interpretation of Higgs cross section measurements. Besides the hard scattering processes, the measurement of different observables of the accompanying underlying event (UE) is essential to test and improve the modelling of Monte Carlo (MC) generators. Furthermore, measurements of QCD processes provide also great confidence in the detector performance and particle reconstruction techniques.

In this note, measurements by the ATLAS experiment [1] at the LHC using proton-proton collision data at a centre-of-mass energy of $\sqrt{s}=7 \mathrm{TeV}$ are presented. Those testing primarily perturbative QCD predictions are briefly summarised in Sec. 2 with results from prompt photon, di-photon and photon plus jet production, inclusive jet and dijet production, jet shapes in top-quark pair events, vector boson plus jets production and $W$ boson plus prompt $J / \psi$ meson production. Finally in Sec. 3, measurements involving soft non-perturbative effects of QCD are briefly summarised with distributions sensitive to the underlying event in QCD jet events followed by results from the $\phi(1020)$ meson production.

\footnotetext{
a e-mail: manuel.proissl@cern.ch
} 


\section{Hard perturbative QCD measurements}

Prompt photons, i.e. not originating from hadronic decays but directly from the hard process or through parton fragmentation, are dominantly produced via the Compton-like $q g \rightarrow q \gamma$ process at LHC and, thus, are valuable probes of the gluon content of the proton with the potential to constrain the gluon PDF. Moreover, they form a considerable background to Standard Model processes such as Higgs boson decays to photon pairs $(H \rightarrow \gamma \gamma)$ and in searches for New Physics. Their production cross section has been measured [2] over a transverse energy range of $100 \mathrm{GeV}<E_{\mathrm{T}}^{\gamma}<1 \mathrm{TeV}$ and a pseudorapidity range of $\left|\eta^{\gamma}\right|<1.37$ (barrel region) and $1.52 \leq\left|\eta^{\gamma}\right|<2.37$ (end-cap region) using a data sample corresponding to an integrated luminosity of $4.6 \mathrm{fb}^{-1}$. The photons are required to be isolated in terms of significant hadronic activity and are selected if the transverse energy $\left(E_{\mathrm{T}}^{\text {iso }}\right)$ within a cone of radius $\Delta R=\sqrt{(\Delta \eta)^{2}+(\Delta \phi)^{2}}=0.4$ centred around the photon in the pseudorapidity and azimuthal angle $(\phi)$ is found to be less than $7 \mathrm{GeV}$. The residual non-prompt background is subtracted using the "two-dimensional side bands" method [3]. The measured differential cross section is compared in Fig. 1 with a next-to-leading order (NLO) QCD calculation including both direct and fragmentation contributions using the JETPHOX 1.3 Monte Carlo program $[4,5]$ based on CT10 [6] and MSTW2008NLO [7] PDF sets. An overall good agreement is observed, except at low $E_{\mathrm{T}}^{\gamma}$, where the contribution from fragmentation photons is dominant [8] and exhibits the importance of higher-order fragmentation terms. Although both shape and uncertainty of the gluon distribution may be constrained by this data over the range $0.03<x<0.3$ (Bjorken $x \sim 2 E_{\mathrm{T}}^{\gamma} / \sqrt{s}$ ), large scale uncertainties of $10-30 \%$ are presently limiting the constraining power.

The need for higher-order predictions has been also demonstrated in the production of isolatedphoton pairs [9]. Their measured cross section is compared to fixed-order NLO (DIPHOX [10] MC program complemented with GAMMA2MC [11]) and next-to-next-to-leading order (NNLO) calculations (2 $\gamma \mathrm{NNLO}$ [12] MC program) and shows significant modelling improvements between the two, although $2 \gamma$ NNLO still slightly underestimates the data since it neglects the contribution from fragmentation photons. Besides that, both fixed-order calculations cannot reproduce the data well at $\Delta \phi_{\gamma \gamma} \simeq \pi$ and low di-photon $p_{\mathrm{T}}$ due to infrared divergences from initial-state soft gluon radiation and missing soft gluon resummation.

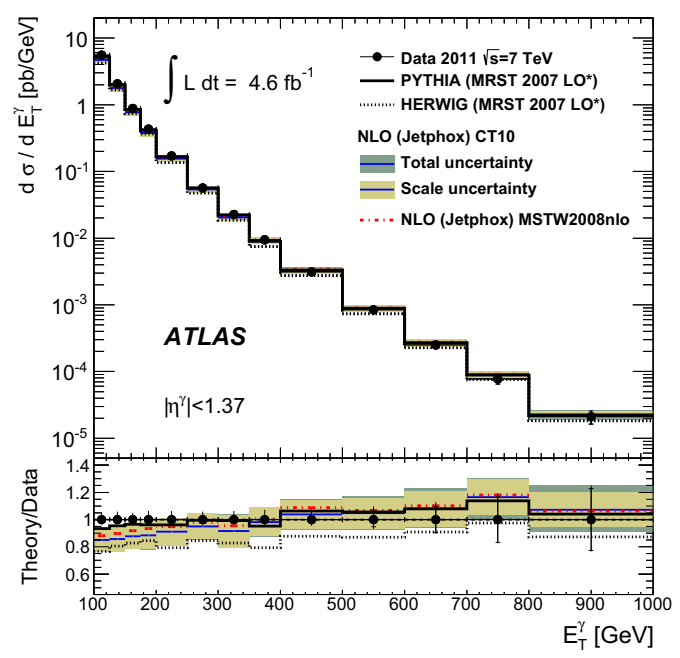

Figure 1. The measured and expected inclusive prompt photon cross section as a function of $E_{\mathrm{T}}^{\gamma}$ [2]. 
QCD@Work 2014

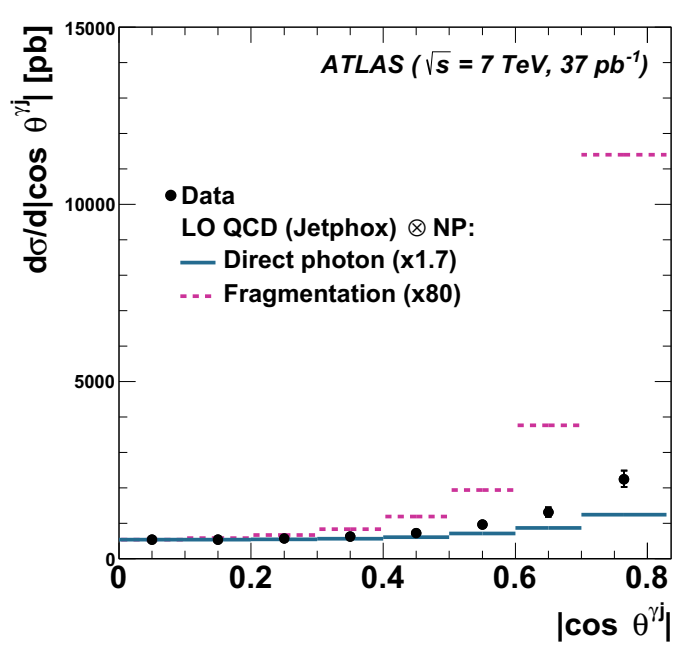

Figure 2. The comparison of the measured cross section for isolated-photon plus jet production with the LO QCD prediction for the direct-photon and fragmentation components [13].

A deeper insight into the fragmentation contributions can be gained from the production of prompt photons in association with a jet, i.e. $p p \rightarrow \gamma+$ jet $+X$ [13]. The angular correlations of the photon plus jet system can be measured to study the dynamics of the hard-scattering process. A measurement using an integrated luminosity of $37 \mathrm{pb}^{-1}$ has been made with a similar photon selection and background rejection as described above. A photon with $E_{\mathrm{T}}^{\gamma}>45 \mathrm{GeV}$ is required to be isolated with $E_{\mathrm{T}}^{\text {iso }}<3 \mathrm{GeV}$ within a $\Delta R<0.4$ cone and accompanied by a non-overlapping jet with $\Delta R(\gamma, \mathrm{jet})>1$, which is reconstructed within the central rapidity range of $\left|y^{\mathrm{jet}}\right|<2.37$ using the anti- $k_{t}$ algorithm [14] with distance parameter $R=0.6$, and has the highest jet transverse momentum $\left(p_{\mathrm{T}}^{\mathrm{jet}}\right)$ in the event with at least $p_{\mathrm{T}}^{\mathrm{jet}}>40 \mathrm{GeV}$. The measured cross section agrees well with NLO QCD predictions, corrected for non-perturbative effects, in a number of angular and scalar observables. The shape of the measured angular distribution $\left|\cos \theta^{\gamma j}\right| \equiv\left|\tanh \left(\Delta y^{\gamma, \text { jet }} / 2\right)\right|$ in Fig. 2 is compared to LO QCD predictions for contributions of the direct and fragmentation processes separately, where the corresponding exchanged particle is either a quark or gluon, respectively. The different behaviour comes from the different spin of the exchanged particle and the better agreement of direct photons with data is therefore consistent with the dominance of quark-exchange processes at the LHC.

Another stringent test of perturbative QCD is the measurement of jet production cross sections. Previously, it has been shown that the combination of ATLAS inclusive jet cross section data with HERA I data reduces the gluon PDF uncertainty at high $x$ [15], and that ratios of jet multiplicities are sensitive to the strong coupling constant $\alpha_{s}$ and theoretical uncertainties can be reduced [16]. Recently, a new measurement has been made of double-differential dijet cross sections as a function of dijet mass $m_{12}<5 \mathrm{TeV}$ and rapidity separation $y^{*}=\left|y_{1}-y_{2}\right| / 2<3.0$ of the two highest $p_{\mathrm{T}}$ jets using an integrated luminosity of $4.5 \mathrm{fb}^{-1}$ [17]. Besides constraining the gluon PDF at high momentum fraction, this measurement is particularly interesting for any dijet resonance searches for physics beyond the Standard Model as new interactions may arise at higher energy scales and induce deviations of expected QCD dijet cross sections at high dijet mass. Since contributions from perturbative (i.e. parton showers, higher-order corrections) and non-perturbative (i.e. hadronization, UE) effects differ for different jet radius definitions, this analysis probes these effects by using anti- $k_{t}$ reconstructed jets with radii, $R=0.4$ and $R=0.6$, which also leaves a distinct signature of different PDF sets used by generators. Fixed-order NLO QCD calculations (NLOJet++ MC program [18]), corrected for both 

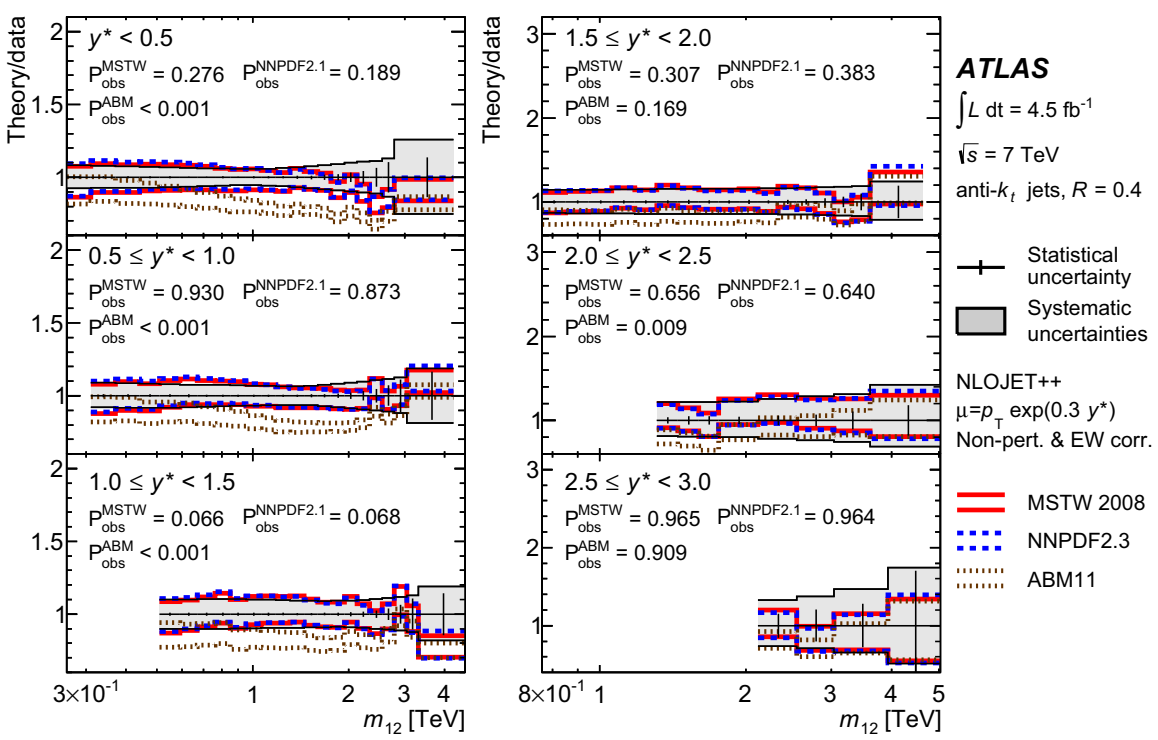

Figure 3. Ratio of the NLO QCD predictions using different PDF sets (MSTW 2008, NNPDF2.3 and ABM11) to the measured double-differential dijet cross section as a function of dijet mass in different $y^{*}$ ranges [17].

non-perturbative and electroweak effects, show for most used PDF sets an overall good agreement with the measured dijet cross section over the full rapidity and dijet mass range considered. As Fig. 3 shows, only the ABM11 PDF set [19] fails to describe the data. Generally, in the $y^{*}<1.5$ range, the absolute cross sections at a particular dijet mass are smaller and the observed p-values resulting from the comparison of theory with data, using a frequentist method based on an asymmetric $\chi^{2}$ definition, are larger when using jets with $R=0.4$ rather than with $R=0.6$.

A better understanding of the internal structure of jets as a function of their $p_{\mathrm{T}}$ is crucial for many Higgs studies or searches for new phenomena, particularly in highly boosted topologies, where substructure methods must be applied. The internal structure of the final reconstructed (merged) jet depends mainly on its originating parton(s). For instance, gluon induced jets are expected to have a broader profile than those induced by quarks, which is due to colour factor differences between $g g g$ and $q q g$ vertices resulting in more parton radiation for gluons. Furthermore, $b$-quark induced jets are expected to be broader than light quark (i.e. $u, d$ ) induced jets. A useful observable to probe the parton shower and hadronization evolution is the jet shape, defined as the normalised $p_{\mathrm{T}}$ flow as a function of the distance to the jet axis. A measurement of $b$-jet shapes has been made in top-quark pair events, dominated by $t \rightarrow W b$, using an integrated luminosity of $1.8 \mathrm{fb}^{-1}$ and achieved a reasonable agreement with predictions from MC generators with the matrix elements calculated up to NLO accuracy [20].

A standard candle of perturbative QCD phenomenology is the production of vector boson plus jets [21]. It is a major background in many resonance searches, such as the $W / Z$ associated $H \rightarrow$ $b \bar{b}$ production [22], and is often used to characterise different phase space regions using, e.g., bins defined by the vector boson $p_{\mathrm{T}}^{V}$. Therefore, an accurate modelling of the $p_{\mathrm{T}}^{V}$ is essential. Initialstate parton radiation and the intrinsic $p_{\mathrm{T}}$ of initial-state partons inside the proton govern the low $p_{\mathrm{T}}^{V}$ range, while the high $p_{\mathrm{T}}^{V}$ range is dominated by quark-gluon scattering. A recent measurement of the $Z$ boson $p_{\mathrm{T}}^{Z}$ cross section [23], using an integrated luminosity of $4.7 \mathrm{fb}^{-1}$, is modelled with perturbative QCD calculations without (FEWZ [24-26], DYNNLO [27, 28] MC programs) and with (ResBos $[29,30] \mathrm{MC}$ program) the resummation of low- $p_{\mathrm{T}}^{Z}$-predominant soft-gluon emissions at next- 

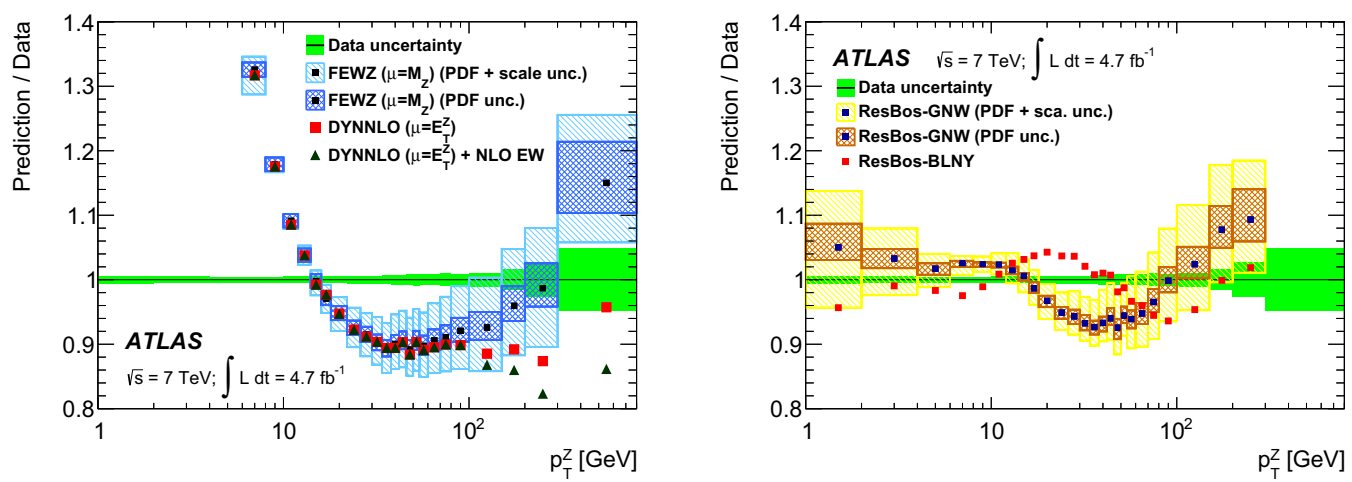

Figure 4. Ratios of QCD predictions without (left) and with (right) soft-gluon resummation to the measured $p_{\mathrm{T}}^{Z}$ distributions [23].

to-next-to-leading-logarithmic (NNLL) accuracy. As shown in Figure 4, the $O\left(\alpha_{S}^{2}\right)$ cross section predictions by FEWZ and DYNNLO diverge as expected at low $p_{\mathrm{T}}^{Z}$ due to the absence of soft-gluon resummation. The choice of the QCD renormalisation and factorisation scales is studied with the DYNNLO program, using both a constant and dynamic scale. The constant scale is set to the $Z$ boson mass $m_{Z}$ and the dynamic scale is set to $E_{\mathrm{T}}^{Z} \equiv m_{Z} \oplus p_{\mathrm{T}}^{Z}$. The shape above $p_{\mathrm{T}}^{Z}>30 \mathrm{GeV}$ agrees better with data when using the dynamic scale, but the normalisation remains about $10 \%$ too low in this region. If, in addition, NLO electroweak corrections are applied, the cross section at high $p_{\mathrm{T}}^{Z}$ drops significantly. On the other hand, the ResBos calculation does not account for the hadronic activity in the event, but as expected agrees much better with the data at low $p_{\mathrm{T}}^{Z}$. The ResBos-BLNY (NLO+NNLL) prediction agrees with the data within 10-12\% and the ResBos-GNW (NNLO+NNLL) prediction achieves an agreement of 5-7\%.

In Higgs boson analyses, such as $H \rightarrow W W$ or $(W / Z) H \rightarrow b \bar{b}$, as well as New Physics searches involving jet signatures, the multiplicity and kinematics of jets are exploited to achieve a better signal from background discrimination. Therefore, a good understanding of the underlying QCD processes is important. The production of a $Z$ boson in association with jets provides a cleaner environment to probe these processes and has been recently measured with $b$-jets using an integrated luminosity of $4.6 \mathrm{fb}^{-1}$ [31]. Perturbative QCD calculations in the four-flavour number scheme (4FNS) consider only
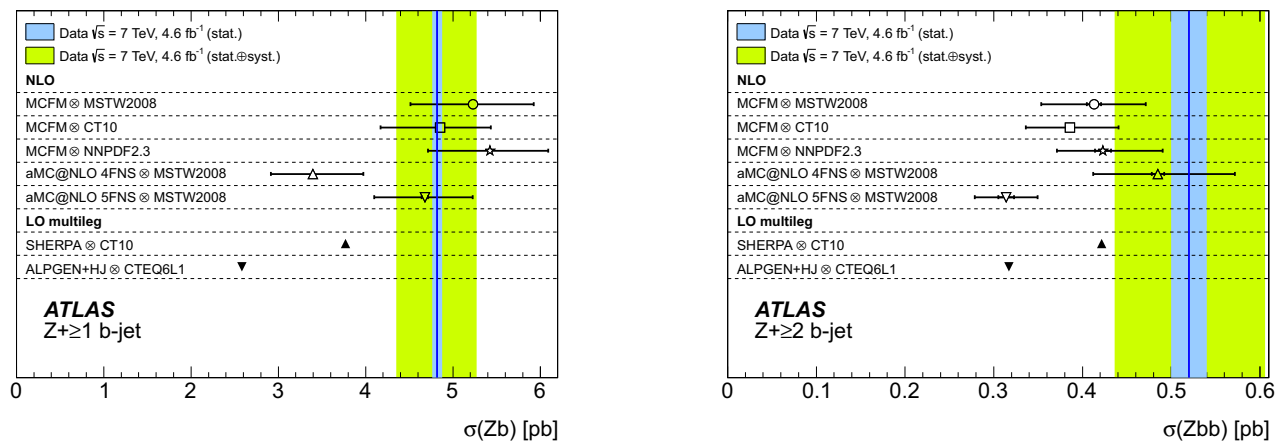

Figure 5. Production cross section measurements for $Z+\geq 1 b$-jet (left) and $Z+\geq 2 b$-jets (right) compared to NLO and LO multi-legged predictions [31]. 
the first two quark generations as initial-state quarks and in the five-flavour number scheme (5FNS) allow also the presence of $b$-quarks in the initial state. If the calculations could be performed to all orders, the results from both schemes should be identical. Figure 5 shows the measured fiducial cross sections for $Z+\geq 1 b$-jet and $Z+\geq 2 b$-jets compared with fixed-order parton-level predictions at NLO in the 5FNS using the MCFM program [32], full particle-level predictions with NLO matrix elements using the aMC@NLO generator [33] and LO multi-legged predictions in the 5FNS using the SHERPA generator [34] and in the 4FNS using the ALPGEN+HERWIG+JIMMY generators [35-37]. While MCFM matches the data within the uncertainties, aMC@NLO underestimates the data in the 4FNS for $Z+\geq 1 b$-jet and in the 5FNS for $Z+\geq 2 b$-jets, while the latter has been only calculated at LO. Both double parton interactions and gluon splitting play an important role in this measurement. For example, all predictions mentioned above underestimate the data at $\Delta R(b, b) \lesssim 1.0$. This may originate from gluon splitting at low angle, i.e. jets associated to two $b$-hadrons.

Another essential vector boson plus jet measurement is the measurement of production of a $W$ boson in association with a single charm quark via the dominant $g s \rightarrow W c$ process, providing an excellent opportunity to constrain the $s$-quark PDF in the proton at $x \sim 0.01$. This measurement has been made using $4.6 \mathrm{fb}^{-1}$ of integrated luminosity [38], with the charm quark tagged through the presence of a soft muon from a semileptonic charm decay within a hadronic jet or through the presence of charged $D^{(*)}$ meson decays. The comparison of these cross section measurements with different PDF sets, interfaced with aMC@NLO, shows that the data favours PDFs with an SU(3)-symmetric light quark sea.

The production of a $W$ boson in association with a prompt $J / \psi$ allows us to test QCD at the perturbative/non-perturbative boundary, sensitive to double parton interactions and provides a new framework to search for physics beyond the Standard Model [39]. Using an integrated luminosity of $4.6 \mathrm{fb}^{-1}$, a yield of $27.4_{-6.5}^{+7.5}$ events has been observed with a statistical significance of $5.1 \sigma$.

\section{Soft non-perturbative QCD measurements}

The underlying event is present in all proton-proton collisions and forms an irreducible background in high precision Standard Model measurements as well as searches for any new physics phenomena. It is therefore crucial to derive better predictions for UE contributions, including multiple parton interactions, QCD colour connections between partons and beam remnants as well as initial and final
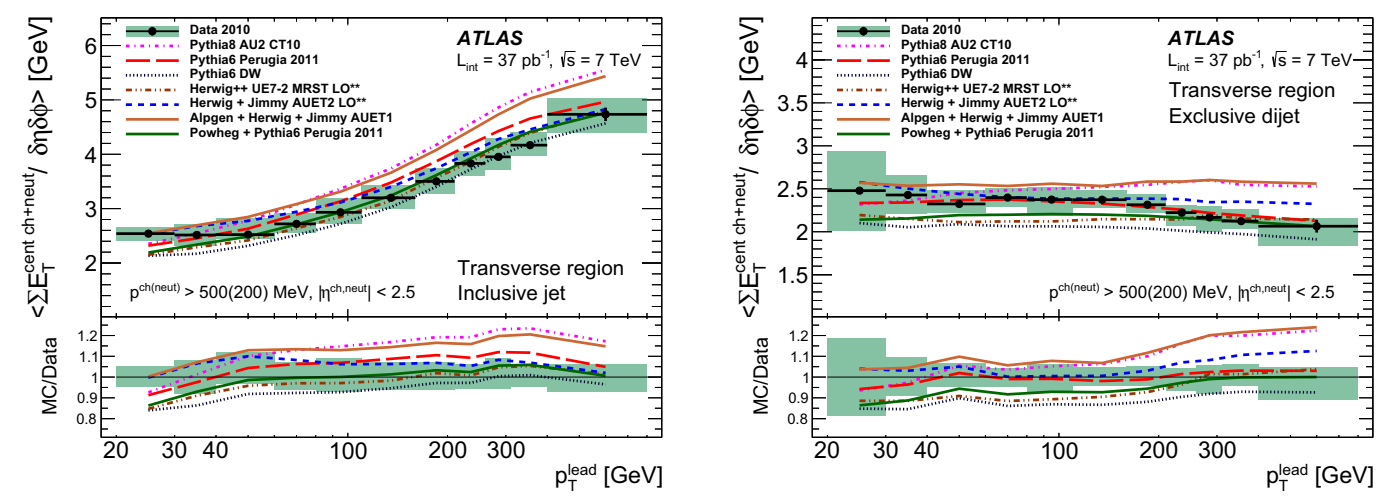

Figure 6. The charged and neutral particle $\sum E_{\mathrm{T}}$ in the transverse region as a function of the leading jet $p_{\mathrm{T}}$ in both inclusive jet (left) and exclusive dijet (right) topologies [40]. 
state radiation. Since the QCD coupling at low scales diverges and, thus, perturbative QCD is not applicable anymore, a tuneable phenomenological approach using MC generators is used. While it is impossible to completely separate UE components from the hard scattering process on an event-level basis, observables that are sensitive to UE properties can be studied. Different observables have been measured in inclusive jet and exclusive dijet events using an integrated luminosity of $37 \mathrm{pb}^{-1}$ [40]. The transverse region, $60^{\circ}<|\Delta \phi|<120^{\circ}$, with respect to the $\phi$ of the highest $p_{\mathrm{T}}$ jet in the event, reconstructed using the anti- $k_{t}$ algorithm with distance parameter $R=0.4$, represents the most sensitive region to probe the UE activity. The charged and neutral particle $\sum E_{\mathrm{T}}$ in this region is shown in Figure 6 as a function of the leading jet $p_{\mathrm{T}}$ and exhibits a rising activity in inclusive jet and nearly flat but slowly decreasing activity in exclusive dijet topologies. An overall good agreement with data was achieved by different tunes of event generators, such as PYTHIA [41, 42].

A useful input to tune MC generators and constrain phenomenological soft hadroproduction models is the measurement of $\phi(1020)$ mesons decaying to opposite sign kaons $\phi \rightarrow K^{+} K^{-}$, which is sensitive to $s$-quark and low- $x$ gluon densities. Recently their production cross section has been measured using an integrated luminosity of $383 \mu \mathrm{b}^{-1}$ [43] with most MC generators failing to describe the data well.

\section{Conclusions}

An extensive set of Standard Model measurements have been made during the LHC Run 1 and generally an excellent agreement with QCD predictions has been achieved. Moreover these measurements have significantly improved our understanding of QCD phenomenology at LHC and have served as an input to constrain PDFs. The discovery of a Higgs boson using the LHC Run 1 dataset has opened the door to a rich precision Higgs physics program. As we will enter a new energy regime with the LHC Run 2, our understanding and modelling of all Standard Model background processes in this high luminosity environment will be paramount, especially for any searches for physics beyond our Standard Model expectation.

\section{Acknowledgements}

On behalf of the ATLAS collaboration, the author thanks CERN for the very successful operation of the LHC in Run 1, as well as the support staff from our institutions without whom ATLAS could not be operated efficiently. The author acknowledges the hard work of the ATLAS Standard Model group for providing a deeper understanding of QCD phenomenology at the LHC. A very special thanks to the organising committee of the QCD @ Work 2014 conference for inviting experts from both theoretical and experimental communities leading to many fruitful discussions in the beautiful setting of Giovinazzo in Bari, Italy.

\section{References}

[1] ATLAS Collaboration, JINST 3, S08003 (2008).

[2] ATLAS Collaboration, Phys. Rev. D 89, 052004 (2014).

[3] ATLAS Collaboration, Phys. Rev. D 83, 052005 (2011).

[4] P. Aurenche, M. Fontannaz, J.-P. Guillet, E. Pilon, and M. Werlen, Phys. Rev. D 73, 094007 (2006).

[5] S. Catani, M. Fontannaz, J.-P. Guillet, and E. Pilon, J. High Energy Phys. 0205, 028 (2002).

[6] H.-L. Lai, M. Guzzi, J. Huston, Z. Li, P. M. Nadolsky, J. Pumplin, and C.-P. Yuan, Phys. Rev. D 82, 074024 (2010). 
[7] A. Martin, W. Stirling, R. Thorne, and G. Watt, Eur. Phys. J. C 63, 189 (2009).

[8] ATLAS Collaboration, ATL-PHYS-PUB-2013-018, https://cds.cern.ch/record/1636863 (2013).

[9] ATLAS Collaboration, J. High Energy Phys. 1301, 086 (2013).

[10] T. Binoth, J. P. Guillet, E. Pilon, and M. Werlen, Eur. Phys. J. C 16, 311-330 (2000).

[11] Z. Bern, L. Dixon, and C. Schmidt, Phys. Rev. D 66, 074018 (2002).

[12] S. Catani, L. Cieri, D. de Florian, G. Ferrera, and M. Grazzini, Phys. Rev. Lett. 108, 072001 (2012).

[13] ATLAS Collaboration, Nucl. Phys. B 875, 483-535 (2013).

[14] M. Cacciari, G.P. Salam, G. Soyez, J. High Energy Phys. 0804, 063 (2008).

[15] ATLAS Collaboration, Eur. Phys. J. C 73, 2509 (2013).

[16] ATLAS Collaboration, ATLAS-CONF-2013-041, https://cds.cern.ch/record/1543225 (2013).

[17] ATLAS Collaboration, J. High Energy Phys. 1405, 059 (2014).

[18] Z. Nagy, Phys. Rev. D 68, 094002 (2003).

[19] S. Alekhin, J. Bluemlein, and S.-O. Moch, PoS LL 2012, 016 (2012).

[20] ATLAS Collaboration, Eur. Phys. J. C 73, 2676 (2013).

[21] ATLAS Collaboration, J. High Energy Phys. 1307, 032 (2013).

[22] ATLAS Collaboration, arXiv:1409.6212 (2014).

[23] ATLAS Collaboration, arXiv:1406.3660 (2014).

[24] K. Melnikov and F. Petriello, Phys. Rev. D 74, 114017 (2006).

[25] R. Gavin, Y. Li, F. Petriello, and S. Quackenbush, Comput. Phys. Commun. 182, 2388-2403 (2011).

[26] Y. Li and F. Petriello, Phys. Rev. D 86, 094034 (2012).

[27] S. Catani, L. Cieri, G. Ferrera, D. de Florian, and M. Grazzini, Phys. Rev. Lett. 103, 082001 (2009).

[28] S. Catani and M. Grazzini, Phys. Rev. Lett. 98, 222002 (2007).

[29] C. Balazs and C. Yuan, Phys. Rev. D 56, 5558-5583 (1997).

[30] M. Guzzi, P. M. Nadolsky, and B. Wang, Phys. Rev. D 90, 014030 (2014).

[31] ATLAS Collaboration, arXiv:1407.3643 (2014).

[32] J. M. Campbell and R. Ellis, Nucl. Phys. Proc. Suppl. 205-206 (2010) 10.

[33] J. Alwall et al., J. High Energy Phys. 1407, 079 (2014).

[34] T. Gleisberg et al., J. High Energy Phys. 0902, 007 (2009).

[35] M. L. Mangano et al., J. High Energy Phys. 0307, 001 (2003).

[36] G. Corcella et al., J. High Energy Phys. 0101, 010 (2001).

[37] J. M. Butterworth, J. R. Forshaw, and M. H. Seymour, Z. Phys. C 72, 637 (1996).

[38] ATLAS Collaboration, J. High Energy Phys. 1405, 068 (2014).

[39] ATLAS Collaboration, J. High Energy Phys. 1404, 172 (2014).

[40] ATLAS Collaboration, Eur. Phys. J. C 74, 2965 (2014).

[41] T. Sjostrand, S. Mrenna, and P. Skands, J. High Energy Phys. 0605, 026 (2006).

[42] T. Sjostrand, S. Mrenna, and P. Skands, Comput. Phys. Commun. 178, 852-867 (2008).

[43] ATLAS Collaboration, Eur. Phys. J. C 74, 2895 (2014). 